\title{
COVID-19 vaccination hesitancy and challenges to mass vaccination
}

\author{
Charlene YC Chau *, MB, BS, MPhil
}

This article was published on 18 Oct 2021 at www.hkmj.org.
Li Ka Shing Faculty of Medicine, The University of Hong Kong, Hong Kong

*Corresponding author: cycchau@connect.hku.hk

Hong Kong Med J 2021;27:377-9

https://doi.org/10.12809/hkmj219639

\section{Introduction}

The suboptimal uptake of vaccines due to vaccine hesitancy remains a pressing global challenge. In Hong Kong, as of August 2021, only 47.4\% and $35.8 \%$ of the population have received the first and second doses of the coronavirus disease 2019 (COVID-19) vaccine, respectively. ${ }^{1}$ This does not satisfy the estimated figure of vaccine uptake (55\%$82 \%)$ to achieve herd immunity against severe acute respiratory syndrome coronavirus 2 (SARS-CoV-2). ${ }^{2}$ However it is important to note that epidemiological (eg, population structures) and immunological factors (eg, waning immunity) will influence the degree of indirect protection conferred by herd immunity. ${ }^{3}$

\section{Determinants of vaccine hesitancy}

Vaccine hesitancy refers to a continuum of behaviours from complete acceptance to refusal. The psychological antecedents that underpin the decision-making process behind vaccine hesitancy could be captured by the $5 \mathrm{C}$ scale: confidence, complacency, collective responsibility, constraints, and calculation. ${ }^{4}$

\section{Confidence}

Confidence is influenced by the trust in vaccines (necessity, effectiveness, and safety), the health system in which a vaccine is delivered, and the policymakers with decision-making power for resource allocation. Surveys report declining vaccination intentions, with variability across countries and socio-economic populations. ${ }^{5}$ Drivers of low confidence are predominantly doubts over the efficacy and long-term effects, and concerns about adverse effects. Possible adverse effects due to vaccine reactogenicity may feed hesitancy; ongoing transparent surveillance systems and effective communication to stakeholders could circumvent this. ${ }^{6}$ Which stakeholder holds the responsibility for legal indemnity against liability for adverse effects has also led to much debate. The COVID-19 vaccine pillar has since announced a no-fault compensation system for vaccine injuries, but the negative impact of this scheme on public perception towards the vaccine requires further exploration. ${ }^{7}$

\section{Collective responsibility}

The strained relationship with mainland China and divided political-cultural identity of Hong Kong citizens undermine collectivism, communal orientation, and empathy, which are positive correlators of collective responsibility. ${ }^{4}$ In particular, distrust in health authorities and political legitimacy may also explain geographical heterogeneity in vaccine intent. 8 The discrepancy of intent rate between China and subpopulations in Hong Kong could stem from the socio-political tensions preceding the outbreak. ${ }^{8,9}$

\section{Complacency}

Intention to take the vaccine in Hong Kong may be related to complacency, notably due to the initial successes in curbing COVID-19 epidemic in Hong Kong, and past experiences with the 2003 SARS epidemic with strict infection control measures. A cross-sectional online survey by Kwok et $\mathrm{al}^{9}$ investigating the uptake rate of COVID-19 vaccine among nurses demonstrated a drop in intention correlated with fewer confirmed cases.

\section{Constraints}

Constraints denote the structural and psychological barriers to availability, affordability, and accessibility. The launch of new vaccination centres, the recruitment of healthcare professionals to administer the vaccine, and walk-in vaccination schemes have targeted structural barriers. Psychological barriers that render inoculation as inconvenient remain a predictor of vaccine hesitancy in Hong Kong, although their effect is non-significant. .,10 $^{2}$

\section{Calculation}

High calculation, that is, a higher perceived risk related to vaccination than infection, leads to non-vaccination possibly due to widely available anti-vaccination materials. ${ }^{4}$ Kwok et al ${ }^{9,10}$ noted that calculation was not a significant predictor of COVID-19 vaccine hesitancy in Hong Kong. However, these studies were conducted in the early phase of the epidemic when limited information on the vaccines was available. 


\section{Strategies to address COVID-19 vaccine hesitancy}

\section{Mandatory vaccination}

Against the backdrop of increasing mortality and the emergence of mutant strains, the radical enactment of mandatory vaccination laws may be considered. Although this may lead to rapid populationwide vaccine uptake, this coercive strategy has its disadvantages. First, it may incite public backlash, erode public trust in governments and health systems, and exacerbate negative vaccination attitudes. The reinvigorated antivaccine movement may affect the uptake of other routine immunisations, which may threaten the re-emergence of previously eradicated diseases..$^{11,12}$ Second, there is an increased financial and administrative burden due to costs in procurement, enforcement, and monitoring. This may not be feasible for some countries with weakened health and economic systems due to the COVID-19 outbreak. More flexible mandates can also be considered, including an opt-out approach without penalty. Yet, exemptions based on personal or philosophical objections may be exploited by

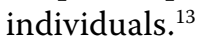

\section{Education and awareness}

Less peremptory strategies can be adopted, including improving public education and raising awareness. In the prevailing anti-vaccination rhetoric surrounding COVID-19, it is important to address the concerns and knowledge gap of the general public. Misinformation about adverse effects and conspiracy theories must be reduced and separated from factual evidence. Technology-based health literacy and media engagement have been shown to alter vaccination acceptance and health-seeking behaviour paradigms. ${ }^{14}$ Nonetheless, this may be a double-edged sword given the vast anti-vaccination misinformation and disinformation available on social media. To combat vaccine hesitancy, strategies to target anti-vaccination propaganda are required. These may include mandates to remove antivaccination content from social media platforms, which could be challenging in countries with strong freedom of speech laws, ${ }^{14}$ or coordinated efforts to address the sources of disinformation campaigns.

\section{Incentives}

Incentive-based approaches have also gained traction globally. The Hong Kong SAR Government has supported financial incentives, from shopping and dining vouchers to lotteries, executive cars and apartments, and social incentives, including permitting access to certain activities or relaxing some social-distancing restrictions only for vaccinated people. Although they may increase vaccination rates in the short term, financial incentives may not be sustainable in the long run. First, they may alter public perception of the vaccine as more dangerous and ineffective, thus lowering vaccine intentions. Second, they may set a precedence for booster vaccinations and other optional vaccinations. Third, they do not always address the core reasons for vaccine hesitancy, which, in the case of Hong Kong, are predominantly effectiveness and safety concerns. ${ }^{15}$

\section{Non-pharmaceutical interventions}

Vaccine or not, non-pharmaceutical interventions (NPIs) remain critical. In Hong Kong, NPIs have been successful in curbing previous and current pandemics, and have included risk communication, travel restrictions, quarantine, and isolation. ${ }^{16}$ Early detection and population screening have been implemented with initial successes, and continue to be optimised. ${ }^{16}$ New molecular virological techniques, such as recombinase polymerase amplification, clustered regularly interspaced short palindromic repeats, and microfluidics have allowed more accurate and rapid diagnosis, allowing for timely isolation. ${ }^{17}$ With the mass rollout of vaccination, two key questions regarding NPI implementation below should be considered.

\section{When can non-pharmaceutical interventions be relaxed or halted?}

Early relaxation of NPIs has been shown to precipitate a rebound in transmission..$^{18}$ However, strict enforcement of NPIs is unlikely to be sustainable over time and carries substantial socioeconomic consequences. Studies have suggested that high vaccination coverage when NPIs are relaxed has a greater reduction of infections than does higher vaccine efficacy. ${ }^{19}$ Nonetheless, the extent of vaccination coverage to be determined as sufficient to reduce the epidemic peak depends on factors such as vaccine efficacy, population characteristics, and virus transmission dynamics. ${ }^{19}$ The NPIs should be relaxed incrementally with stringent disease surveillance, and re-introduced with an increase in case numbers.

\section{Which non-pharmaceutical interventions can be relaxed?}

Risk communication interventions are the most effective in reducing case spreading and should be maintained. ${ }^{20,21}$ These include government actions to educate and advise the general public, and effectively links other NPIs. For instance, travel warnings may influence travel restriction measures, whereas information campaigns about transmission routes of SARS-CoV-2 may impact social distancing measures. The less coercive and costly nature of this 
theme of NPIs may also promote better compliance and implementation. The nature and type of NPIs to be relaxed should be tailored to individual countries and adjusted according to the evolving epidemic situation (eg, reproduction number of COVID-19, the emergence of new strains), with support given to impacted populations and industries. ${ }^{16}$ In addition, the temporal distribution and the diversity of NPIs should be considered considering the interdependence of all interventions. ${ }^{20}$ Lifting all interventions at the same time should be avoided to prevent second epidemic waves.

\section{Conclusion}

Mass rollout of the COVID-19 vaccination should take into consideration the psychological antecedents of vaccine hesitancy. Other measures including education and NPIs should also be explored in a synergistic effort to end the COVID-19 pandemic.

\section{Author contributions}

The author contributed to the design, acquisition of data, analysis of data, drafting of the manuscript, and critical revision of the manuscript for important intellectual content.

\section{Conflicts of interest}

The author has no conflicts of interest to disclose.

\section{Funding/support}

This study received no specific grant from any funding agency in the public, commercial, or not-for-profit sectors.

\section{References}

1. Hong Kong SAR Government. Early vaccination for all. It's more than a jab. 2020. Available from: https://www. covidvaccine.gov.hk/en/. Accessed 1 Aug 2021.

2. Wong MC, Wong EL, Huang J, et al. Acceptance of the COVID-19 vaccine based on the health belief model: a population-based survey in Hong Kong. Vaccine 2021;39:1148-56.

3. Randolph HE, Barreiro LB. Herd immunity: understanding COVID-19. Immunity 2020;52:737-41.

4. Betsch C, Schmid P, Heinemeier D, Korn L, Holtmann C, Böhm R. Beyond confidence: development of a measure assessing the $5 \mathrm{C}$ psychological antecedents of vaccination. PLoS One 2018;13:e0208601.

5. Robinson E, Jones A, Lesser I, Daly M. International estimates of intended uptake and refusal of COVID-19 vaccines: a rapid systematic review and meta-analysis of large nationally representative samples. Vaccine 2021;39:2024-34.

6. Wadman M. Public needs to prep for vaccine side effects. Science 2020;370:1022.

7. Mungwira RG, Guillard C, Saldaña A, et al. Global landscape analysis of no-fault compensation programmes for vaccine injuries: a review and survey of implementing countries. PLoS One 2020;15:e0233334.

8. Hartley K, Jarvis DS. Policymaking in a low-trust state: legitimacy, state capacity, and responses to COVID-19 in Hong Kong. Policy Soc 2020;39:403-23.

9. Kwok KO, Li KK, Wei WI, Tang A, Wong SY, Lee SS. Influenza vaccine uptake, COVID-19 vaccination intention and vaccine hesitancy among nurses: a survey. Int J Nurs Stud 2021;114:103854.

10. Kwok KO, Li KK, Tang A, et al. Psychobehavioral responses and likelihood of receiving COVID-19 vaccines during the pandemic, Hong Kong. Emerg Infect Dis 2021;27:1802-10.

11. Hotez PJ. COVID19 meets the antivaccine movement. Microbes Infect 2020;22:162-4.

12. Hussain A, Ali S, Ahmed M, Hussain S. The antivaccination movement: a regression in modern medicine. Cureus 2018;10:e2919.

13. MacDonald NE, Harmon S, Dube E, et al. Mandatory infant \& childhood immunization: rationales, issues and knowledge gaps. Vaccine 2018;36:5811-8.

14. Wilson SL, Wiysonge C. Social media and vaccine hesitancy. BMJ Global Health 2020;5:e004206.

15. Luk TT, Zhao S, Wu Y, Wong JY, Wang MP, Lam TH. Prevalence and determinants of SARS-CoV-2 vaccine hesitancy in Hong Kong: a population-based survey. Vaccine 2021;39:3602-7.

16. Yu XY, Xu C, Wang HW, et al. Effective mitigation strategy in early stage of COVID-19 pandemic in China. Infect Dis Poverty 2020;9:141.

17. Luo Z, Ang MJ, Chan SY, et al. Combating the coronavirus pandemic: early detection, medical treatment, and a concerted effort by the global community. Research (Wash D C) 2020;2020:6925296.

18. Aravindakshan A, Boehnke J, Gholami E, Nayak A. Preparing for a future COVID-19 wave: insights and limitations from a data-driven evaluation of nonpharmaceutical interventions in Germany. Sci Rep 2020;10:20084.

19. Patel MD, Rosenstrom E, Ivy JS, et al. Association of simulated COVID-19 vaccination and nonpharmaceutical interventions with infections, hospitalizations, and mortality. JAMA Netw Open 2021;4:e2110782.

20. Chan LY, Yuan B, Convertino M. COVID-19 nonpharmaceutical intervention portfolio effectiveness and risk communication predominance. Sci Rep 2021;11:10605.

21. Haug N, Geyrhofer L, Londei A, et al. Ranking the effectiveness of worldwide COVID-19 government interventions. Nat Hum Behav 2020;4:1303-12. 